\title{
Parameter Identification of Capacitive Power Transfer System Based on Spectrum Analysis
}

\author{
Chen Chen, Chenhui Li, Chenglin Liao, and Lifang Wang
}

\begin{abstract}
Capacitive power transfer (CPT) technology is a newly emerging research focus for EV charging applications. Due to the absence of eddy current loss and light weight of the capacitive coupling metal plates, CPT technology is considered to be a promising alternative to the inductive power transfer (IPT) technology. However, the parameter identification of the CPT system has not been well studied. In this paper, a multi-parameter identification method based on spectral information is proposed, which based on rational fractional fitting algorithm and network synthesis theory. By adding some known parameters to this method, the parameter identification algorithm under constraint is proposed to improve the noise tolerance of the identification results, and at the same time, it can also have better identification accuracy. The experimental results show that the parameter identification algorithm based on spectrum information can effectively identify the parameters of the circuit model of the CPT system, and the identification accuracy is less than $10 \%$.
\end{abstract}

Index Terms - Capacitive power transfer, frequency spectrum, network synthesis, noise tolerance, parameter identification.

\section{INTRODUCTION}

$\mathrm{C}$ APACITIVE power transfer (CPT) [1], [2] technology transfers energy through the electric field between the air gap. This idea can be traced back to Nikola Tesla's bold idea of transferring wireless energy through the capacitance of the ionosphere. Recently, with the increase of the power capacity of the CPT system, it has become a new research focus in the field of wireless charging for electric vehicles.

CPT technology has several unique advantages against the widely-used inductive power transfer (IPT) technology. First, the electric fields do not generate eddy current in the metal material nearby as the magnetic fields do. Besides, the capacitive coupling is usually composed of several conductive plate pairs with no ferrite required, so the coupling part of the CPT is in low cost and light in weight [3]. With these advantages, CPT is considered to be a promising technology in wireless EV charging [4].

Jiejian Dai proposed a design composed of conducting foil at the rear of the EV and a foam-based bumper at the charging station. This technique allows for simple mechanical implementation and avoids the pitfalls associated with alignment and air

Manuscript received July 26, 2018. (Corresponding author: Chenglin Liao.)

The authors are with the Key Laboratory of Power Electronics and Electric Drive, Institute of Electrical Engineering, Chinese Academy of Sciences, Beijing 100190, China (e-mail: chenchen@mail.iee.ac.cn; lichenhui@mail.iee. ac.cn; liaocl@mail.iee.ac.cn; wlf@mail.iee.ac.cn).

Digital Object Identifier 10.24295/CPSSTPEA.2018.00017 gap maintenance usually encountered with CPT systems [5].

The double-sided LCLC CPT system proposed by Lu is a breakthrough in designs of high-power long-distance CPT system, and this prototype system can transfer $2.4 \mathrm{~kW}$ power at a distance of $150 \mathrm{~mm}$. This work provides a complete design of CPT system for EV charging which reaches a DC-to-DC efficiency of over $90 \%$ and works on a relatively low frequency of $1 \mathrm{MHz}$ [6].

$\mathrm{Lu}$ also refined LCLC compensation to CLLC compensation, which reduces the inductance needed for compensation [7]. A simpler LC compensation is also analyzed comparing with the LCLC topology which seemed to be more feasible as it has less components to tune [8]. Due to its simplicity, it is more likely to be realized on EV charging scenario [9].

However, during the wireless charging process of EV, wireless power transfer (WPT) system often needs to work under different coupling conditions due to the uncertainty of the placement position and external conditions of the coupling device. Therefore, the same transmitter-end device may need to have the ability to supply power to multiple receiver devices with different parameters. At present, many parameter identification methods have been developed in inductively coupled power transfer (ICPT) systems, but there are few studies on the parameter identification of the CPT system [10].

In the parameter identification study of ICPT system, most of the identification algorithms are only for a single unknown parameter under the premise of known circuit model parameters. Reference [11] is based on the principle of energy conservation, using the circuit model of the matching circuit to derive the relationship between the voltage and current at the output of the inverter and the load at the receiving end, so as to realize parameter identification of the load at the output. The reference [12] realizes the parameter identification of the output load by measuring the decay speed of the circuit's free oscillation.

For the case where the coupled inductance and the load resistance are unknown, the reference [12] uses the standard load resistance at the output end. First, the coupled inductor is identified under the operating condition of the standard load, and then the actual load resistance is performed under the condition that the coupled inductor identification value is obtained, thereby achieving multi-parameter identification.

In the ICPT system, the change of the coupled inductance does not affect the phase of the reflected impedance. The reference [13] measures the voltage and current output from the inverter, and uses the phase of the reflected impedance to identify the load resistance, and then uses the reflection im- 
pedance amplitude to identify the coupled inductance. This realizes the identification of coupled inductance and output load.

The identification methods used in the above ICPT system often rely on accurate measurement of known parameters of the circuit or directly interpolate fitting results directly from the experimental results. Each identification method is often designed independently according to a specific identification target, and the unknown parameter is also limited to the coupled inductance and the load resistance.

At present, the parameter identification of the CPT system is not well study, this is due to its higher operating frequency and higher requirements on the sampling circuits. On the one hand, there is a coupling relationship between the coupling capacitance and the reflected impedance of CPT, which leads us to be unable to use the method mentioned in reference [13]. On the other hand, the resonant operating frequency of the capacitive system is narrower, and its working state is greatly affected by the matching parameters, if the circuit parameters cannot be accurately measured, it is difficult to obtain good estimation accuracy by using the method described in the reference [12].

Therefore, this paper proposes a more general parameter identification algorithm for the CPT system. Since the result obtained by directly using the driving-point function is very sensitive to noise, in order to improve the applicability of parameter identification, the partial loss resistance and matching circuit component parameters in the model circuit are taken as known values, and by adding these known values as constraints to the identification system model, the noise tolerance of the algorithm can be improved.

\section{Parameter Identification Method Using Frequency SPECTRUM INFORMATION}

The circuit model of CPT system is shown in Fig. 1, where $\mathrm{L}_{1}, \mathrm{C}_{1}, \mathrm{~L}_{2}, \mathrm{C}_{2}$ respectively for the transmitter and receiver matching circuit inductance and capacitance, $\mathrm{Cm}$ for the coupling capacitor, $\mathrm{R}_{\text {load }}$ for the load resistance. By analyzing its circuit model, we can know that when the output load is stable, the system can be seen as a single-port network driven by the inverter output, and the internal components of the network are lumped-parameter linear components. Therefore, the problem of parameter identification for CPT systems can be abstracted as a parameter identification problem for a single-port network. According to the circuit network theory, for a linear single-port network, its external characteristics are fully determined by the driving-point function of the port, the driving-point function can select admittance functions or impedance functions [14]. Here, taking the admittance function as an example, the driving-point function can be expressed in a rational fractional form:

$$
Y(\mathrm{~s})=\frac{I_{i n}(\mathrm{~s})}{U_{\text {in }}(\mathrm{s})}=\frac{\sum a_{i} s^{i}}{\sum b_{j} s^{j}}
$$

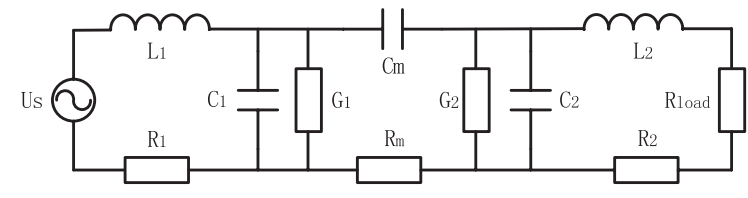

Fig. 1. Circuit model of CPT system.

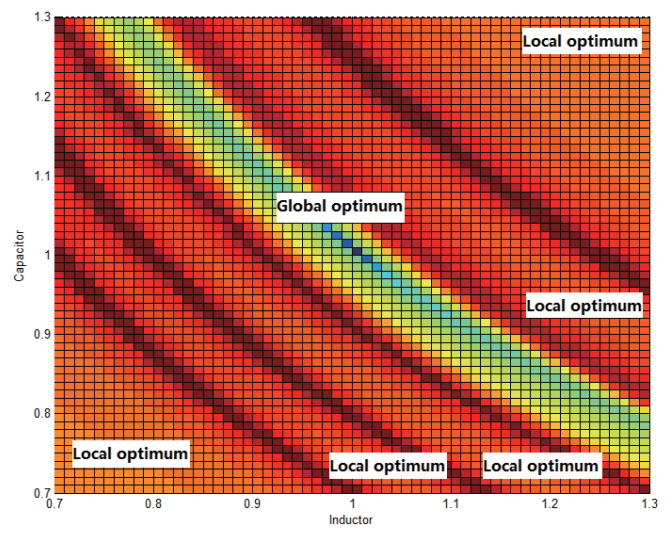

Fig. 2. Solution space map.

where, $\mathrm{I}_{\text {in }}$ and $\mathrm{U}_{\text {in }}$ represent the input current and voltage of the network port respectively, the parameters $a$ and $b$ in the above equation are real numbers, and the order of the numerator and denominator is determined by the topology and component parameters of the circuit.

Next, we need to fit the finite discrete points you have collected, it can generally be assumed that the noise distribution of the sampled values follows a normal distribution, and the sum of the squares of the fitting errors can be used as a criterion.

Since the fitting function is a nonlinear function, if the network is fitted directly using the second-order error minimum principle, the nonlinear least squares problem will be obtained.

$$
\min \left\|\tilde{Y}\left(\mathrm{j} \omega_{i}\right)-Y\left(\mathrm{j} \omega_{i}\right)\right\|_{2}
$$

where $\tilde{Y}(\mathrm{~s})=\frac{\sum k_{i} s^{i}}{\sum d_{j} s^{j}}$ means the fitted function, $k_{i}$ and $d_{j}$ are the undetermined coefficients of the function.

Directly using the sum of squared errors as an optimization index, the optimization problem is that the function is not non-convex, and the fitting error has multiple local optima in the search space, as shown in Fig. 2. Therefore, directly using ordinary nonlinear optimization algorithms, such as the Levenberg-Marquardt method or the trust region method, it is difficult to converge to the optimal value for nonlinear optimization.

In order to solve the problem of a locally optimal solution of nonlinear least squares fitting algorithm in rational fractional fitting, we use the Vector Fitting algorithm [15] to solve the nonlinear least squares problem. First, transform $\tilde{Y}(\mathrm{~s})$ in (2) into a fractional representation by partial fractionation, 


$$
\tilde{Y}(\mathrm{~s})=\frac{\sum\left(\mathrm{s}-\mathrm{z}_{i}\right)}{\sum\left(\mathrm{s}-\mathrm{p}_{j}\right)}
$$

then multiplied by the weight $\frac{\sum\left(\mathrm{s}-\mathrm{p}_{i}\right)}{\sum\left(\mathrm{s}-\tilde{\mathrm{p}}_{i}\right)}$ to get the linearized least squares problem, in the formula, $z_{i}$ and $p_{j}$ represent the zero point and the pole, respectively, and $\tilde{p}_{i}$ is expressed as the estimated pole.

$$
\min \left\|(\sigma \tilde{Y})\left(\mathrm{j} \omega_{i}\right)-Y\left(\mathrm{j} \omega_{i}\right) \sigma\left(\mathrm{j} \omega_{i}\right)\right\|_{2}
$$

where $(\sigma \tilde{Y})(\mathrm{s})=\frac{\sum\left(\mathrm{s}-\mathrm{z}_{i}\right)}{\sum\left(\mathrm{s}-\mathrm{p}_{j}\right)}, \sigma(\mathrm{s})=\frac{\sum\left(\mathrm{s}-\mathrm{p}_{i}\right)}{\sum\left(\mathrm{s}-\tilde{\mathrm{p}}_{i}\right)}$. Therefore, the Vector Fitting algorithm can be used to fit the driving-point function. After using the Vector Fitting algorithm to fit the system's driving-point function, we need to find actual circuit parameters from the function expression. In the CPT system, since the topology of the matching circuit is mostly a ladder network structure, the Cauer synthesis method [16] can be used to get the synthesis of the circuit network. The driving-point function expression of the system regarding the circuit component is obtained as follows:

$$
Y(\mathrm{~s})=\frac{1}{L_{1} s+R_{1}+\frac{1}{C_{1} s+G_{1}+\frac{1}{\frac{1}{C_{m} s}+R_{m}+\frac{1}{C_{2} s+G_{2}+\frac{1}{L_{2} s+R_{2}+R_{\text {load }}}}}}}
$$

Expand (5) to get the admittance function expression of the (1), the highest order of the obtained molecular terms is 4 times, and the denominator term is 5 times, as shown in (6).

$$
Y(s)=\frac{a_{4} s^{4}+a_{3} s^{3}+a_{2} s^{2}+a_{1} s+a_{0}}{b_{5} s^{5}+b_{4} s^{4}+b_{3} s^{3}+b_{2} s^{2}+b_{1} s+b_{0}}
$$

Using Vector Fitting algorithm as a rational fractional fitting algorithm, Cauer synthesis method is used as a network synthesis method to identify the parameters in the circuit, then the correlation coefficients in (6) can be obtain derived, which means that the effective parameters of the actual circuit are obtained.

To sum up, the input parameter identification of the CPT system consists of two parts:

1) Fitting of driving-point function. By applying the excitation and measuring the system response, the external characteristics of the network in the form of the driving-point function are obtained by fitting.

2) Integration of network parameters. According to the expression of the driving-point function, the realization of the circuit network in the corresponding topology is obtained, take this implementation value as an estimate of the parameter.

Through simulation, the parameter identification results under noiseless sampling are shown in TABLE I.

According to the simulation experiment under the noise, if
TABLE I

Noiseless Lossy Model Identification Results

\begin{tabular}{ccc}
\hline \hline Circuit Parameters & $\begin{array}{c}\text { Loss Model } \\
\text { Identification Results } \\
\text { When } \mathrm{R}_{\mathrm{m}}=100 \Omega\end{array}$ & Model Parameters \\
\hline $\mathrm{L}_{1}(\mathrm{H})$ & $8.2240 \mathrm{e}-005$ & $82.24 \mathrm{uH}$ \\
$\mathrm{R}_{1}(\Omega)$ & 3.4401 & $3.44 \Omega$ \\
$\mathrm{C}_{1}(\mathrm{~F})$ & $2.8800 \mathrm{e}-010$ & $288 \mathrm{pF}$ \\
$\mathrm{G}_{1}(\mathrm{~S})$ & $1.0000 \mathrm{e}-005$ & $10 \mathrm{e}-6 \mathrm{~S}$ \\
$\mathrm{C}_{\mathrm{m}}(\mathrm{F})$ & $1.9995 \mathrm{e}-011$ & $20 \mathrm{pF}$ \\
$\mathrm{R}_{\mathrm{m}}(\Omega)$ & $1.0003 \mathrm{e}+002$ & $100 \Omega$ \\
$\mathrm{C}_{2}(\mathrm{~F})$ & $2.8801 \mathrm{e}-010$ & $288 \mathrm{pF}$ \\
$\mathrm{G}_{2}(\mathrm{~S})$ & $9.9966 \mathrm{e}-006$ & $10 \mathrm{e}-6 \mathrm{~S}$ \\
$\mathrm{~L}_{2}(\mathrm{H})$ & $8.2277 \mathrm{e}-005$ & $82.24 \mathrm{uH}$ \\
$\mathrm{R}_{\text {load }}+\mathrm{R}_{2}(\Omega)$ & 36.5561 & $36.54 \Omega$ \\
\hline \hline
\end{tabular}

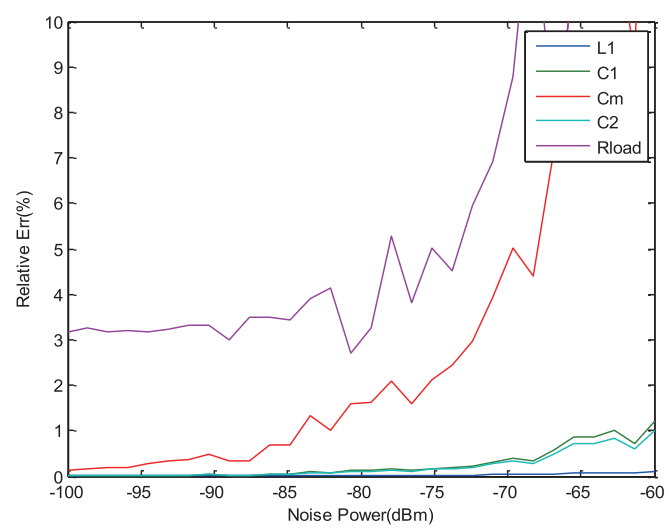

Fig. 3. Identification error of partial lossless model under different sampling noise power.

we want to achieve $10 \%$ estimation error in the experiment, the sampled value noise needs to be higher than $70 \mathrm{dBm}$, as shown in Fig. 3, where shows the relationship between the error rate of the identification parameters and the noise. It can be clearly seen from the Fig. 3 that the sensitivity of $\mathrm{Cm}$ and $\mathrm{R}_{\text {load }}$ to noise is higher than other parameters, and once the noise exceeds $70 \mathrm{dBm}$, the identification error rate of $\mathrm{R}_{\text {load }}$ will exceed $10 \%$.

\section{Parameter Identification Algorithm Under CONSTRAINTS}

Taking a CPT system with double-sided LC matching as an example, assuming that the inductance L1, L2, coupling capacitance $\mathrm{Cm}$, and load resistance $\mathrm{R}_{\mathrm{load}}$ in the circuit parameters are unknown, the circuit model is shown in Fig. 4. The transmitter resistor and capacitor parameters are known to form a two-port circuit $\mathrm{Np}$, the receiver resistor and capacitor parameters are known to form a two-port circuit Ns.

In this algorithm, the unknown variables are modeled in a two-port network, the circuit is decomposed according to the unknown part and the known part, and the unknown parts are separately solved in an iterative manner, and at the same time, the rational fractional fitting method is used, the fol- 


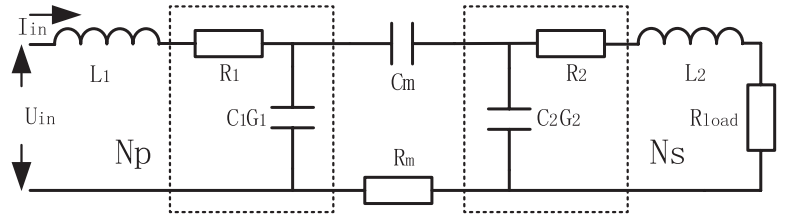

Fig. 4. The parameter identification model under the constraint of double-sided LC matched CPT system.

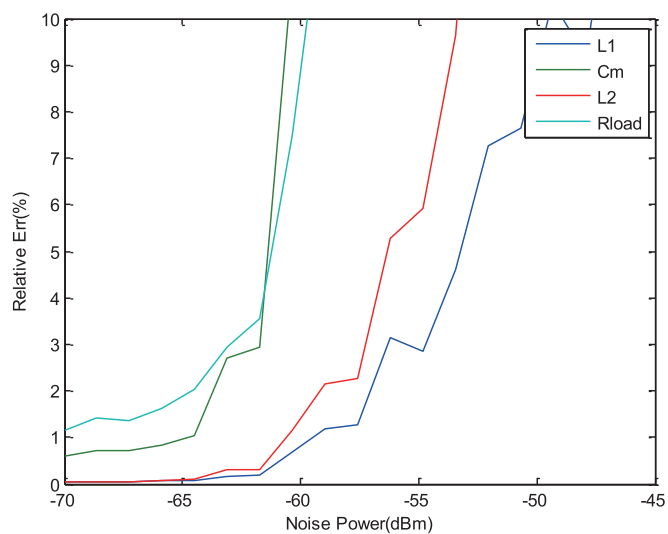

Fig. 5. Identification error of identification algorithm under constraint with different sampling noise power.

lowing fitting algorithm can be designed.

1) Given the initial value of load resistance and coupling capacitance $\tilde{R}_{\text {load }}, \tilde{C}_{m}$ and the inductance $\tilde{l}_{1}, \tilde{l}_{2}$ at both ends.

2) Taking $\tilde{R}_{\text {load }}, \tilde{C}_{m}$ and $\tilde{l}_{2}$ as known variables, the back-end circuit of the inductor $l_{1}$ is known, and the inductor $l_{1}$ is fitted and solved under the back-end constraint as a new front-end inductance estimated value $\tilde{l}_{1}=l_{1}$.

3) Taking $\tilde{R}_{\text {load }}, \tilde{l}_{1}$ and $\tilde{l}_{2}$ as known variables, the front and back-end circuits of the coupling capacitor $C_{m}$ are known, and the coupling capacitor $C_{m}$ is fitted and solved under the front and rear end constraints as a new coupling capacitor estimate $\tilde{C}_{m}=C_{m}$.

4) Taking $\tilde{l}_{1}$ and $\tilde{C}_{m}$ as known variables, the front-end circuits of the inductor and load resistors $l_{2}$ and $R_{\text {load }}$ are known, and the inductance and load resistance $l_{2}$ and $R_{\text {load }}$ are fitted and solved under the front-end constraints as a new coupling capacitor estimate $\tilde{l}_{2}=l_{2}, \tilde{R}_{\text {load }}=R_{\text {load }}$.

5) If the fitting error is less than the threshold, it ends, otherwise, it returns to 2).

In the above algorithm, by modeling the unknown variables in the form of a two-port network, the circuit is decomposed according to the position part and the known part, and the unknown parts are separately solved in the form of iterations.

Though the simulation experiment under the noise, we can see that if we want to achieve $10 \%$ estimation error in the experiment, the sample value noise needs to be higher than $60 \mathrm{dBm}$, as shown in Fig. 5, compared to the identification algorithm based on spectrum information without constraint, the new algorithm can reduce signal-to-noise ratio require-

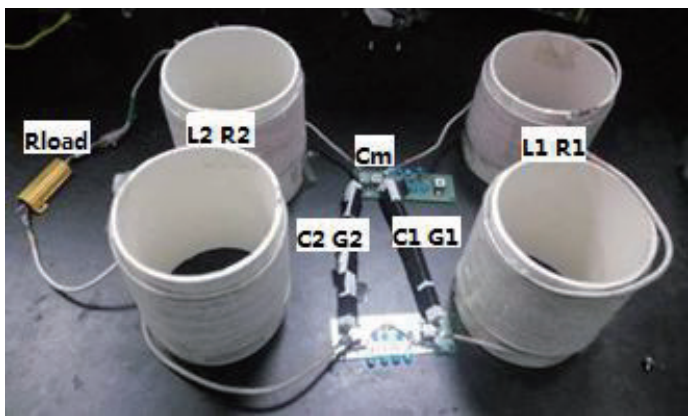

Fig. 6. Double-sided LC matched CPT system model circuit physical map.

TABLE II

Parameter Identification Effect of Parameter Identification Algorithm on Model Physical Circuit

\begin{tabular}{ccc}
\hline \hline Circuit Parameters & $\begin{array}{c}\text { Constraint Model } \\
\text { Identification Results }\end{array}$ & $\begin{array}{c}\text { Component } \\
\text { Measurement }\end{array}$ \\
\hline $\mathrm{L}_{1}$ & $7.6887 \mathrm{e}-005$ & $77.1773 \mathrm{uH}$ \\
$\mathrm{R}_{1}$ & - & $3.6928 \Omega$ \\
$\mathrm{C}_{1}$ & - & $265.7394 \mathrm{pF}$ \\
$\mathrm{G}_{1}$ & - & $5 \mathrm{e}-6 \mathrm{~S}$ \\
$\mathrm{C}_{\mathrm{m}}$ & $2.0501 \mathrm{e}-011$ & $20 \mathrm{pF}$ \\
$\mathrm{R}_{\mathrm{m}}$ & - & - \\
$\mathrm{C}_{2}$ & - & $252.2711 \mathrm{pF}$ \\
$\mathrm{G}_{2}$ & - & $6 \mathrm{e}-6$ \\
$\mathrm{~L}_{2}$ & $7.8756 \mathrm{e}-005$ & $78.8456 \mathrm{uH}$ \\
$\mathrm{R}_{2}$ & - & $3.43 \Omega$ \\
$\mathrm{R}_{\text {load }}$ & 20.9163 & $20 \Omega$ \\
\hline \hline
\end{tabular}

ments of approximately $10 \mathrm{~dB}$.

\section{Model Circuit Parameter Identification EXPERIMENT}

In order to verify the applicability of the algorithm in the actual circuit parameter identification. According to the circuit model of the double-sided LC matching coupling capacitor shown in Fig. 1, the circuit physical map is shown in Fig. 6. The electronic system is used to build the circuit system and the equivalent model physical circuit is made.

A hollow flat wound coil made of 600 strands x $0.1 \mathrm{~mm}$ Litz wire is used in the circuit as an inductor in the matching circuit. A plurality of EPCOS film capacitors with a capacitance of $1 \mathrm{nF}$ are used in series as the capacitance in the matching circuit, and the coupling capacitance is replaced with a $15 \mathrm{pF}$ ceramic capacitor. The actual parameters of the above components were measured using an Agilent E4980A LCR digital bridge, and the actual parameters of the system were as shown in the measured values of the components in TABLE II.

Comparing the constraint model fitting results with the measurement results, the constraint model is constrained by the measured values of the known components, and the identification results are close to the actual circuit parameters, as shown in Fig. 7, where the red line is the result of the vector network analyzer measurement, while the green line 

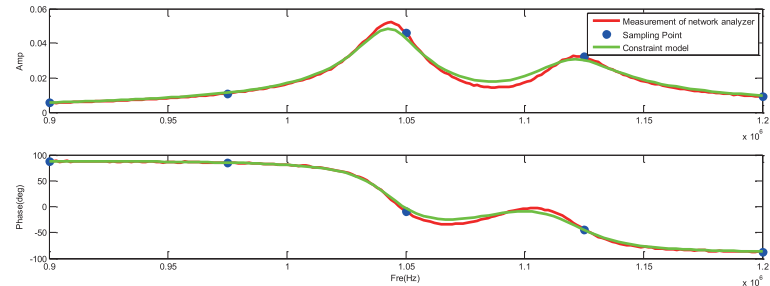

Fig. 7. Comparison of the results of the constraint model fitting and the measurement results.
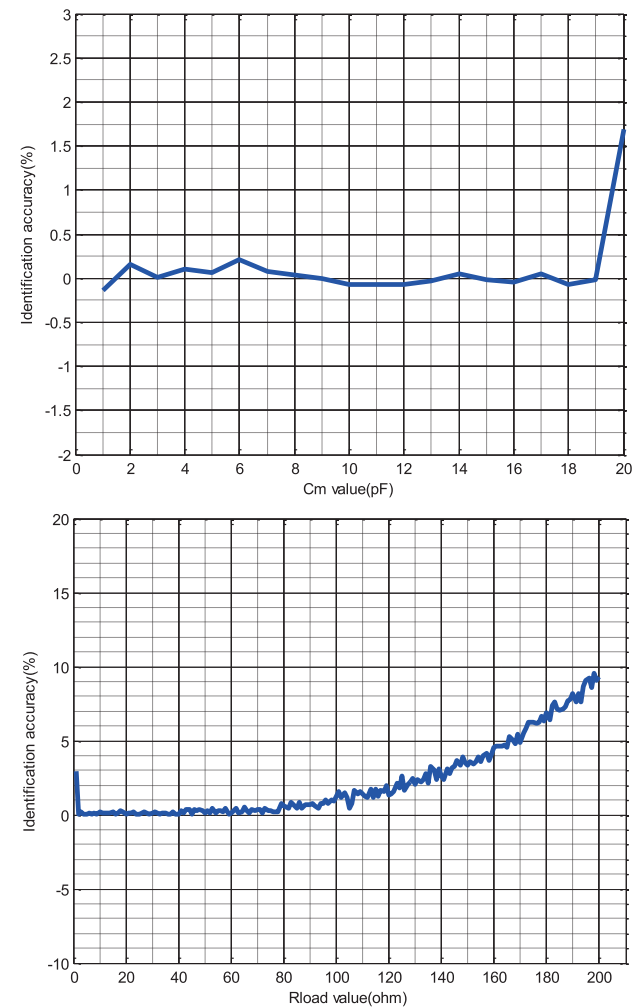

Fig. 8. (a) Relationship between coupling capacitance change and identification accuracy. (b) Relationship between load resistance change and identification accuracy.

is the result of fitting the five blue sampling points using our proposed identification algorithm. It can also be seen from TABLE II that the constraint model can accurately identify the value of the circuit component and the identification accuracy is less than $10 \%$. At the same time, the noise is set to $65 \mathrm{dBm}$. By changing the value of the load resistance and keeping the other parameters of the circuit unchanged, a curve about the load value and the identification accuracy can be obtained, as shown in Fig. 8(b). Keeping the noise at $65 \mathrm{dBm}$, by changing the value of the coupling capacitor and keeping the other parameters of the circuit unchanged, a curve about the coupling capacitance value and the identification accuracy can be obtained, as shown in Fig. 8(a).

\section{CONCLUSION}

This paper proposes a method of parameter identification based on spectrum information, by adding known param- eters of the circuit, the accuracy and noise tolerance of the identification algorithm are improved. In order to verify the proposed algorithm, this paper firsts builds a double-sided LC matched CPT system model physical circuit as shown in Fig. 6. Then, the vector network analyzer is used to collect the spectrum information of the circuit, and the parameter identification algorithm is applied on it. The final result verifies that the parameter identification algorithm based on spectrum information can achieve good results, which not only can effectively identify the unknown parameters in the system model, but also achieve the identification result with less than $10 \%$ accuracy.

\section{REFERENCES}

[1] J. T. Boys and G. A. Covic, "The inductive power transfer story at the university of auckland," IEEE Circuits and Systems Magazine, vol. 15, no. 2, pp. 6-27, May 2015.

[2] A. Karalis, J. D. Joannopoulos, and M. Soljačić, "Efficient wireless non-radiative mid-range energy transfer," Annals of Physics, vol. 323, no. 1, pp. 34-48, Jan. 2008.

[3] C. H. Li, X. M. zhao, C. L. Liao, and L. F. Wang, "A graphical analysis on compensation designs of large-gap CPT systems for EV charging applications," CES Transactions on Electrical Machines and Systems, vol. 3, no. 2, pp. 232-242, Jul. 2018.

[4] C. Mi, "High power capacitive power transfer for electric vehicle charging applications," in 6th International Conference on Power Electronics Systems and Applications (PESA), 2015, pp. 1-4.

[5] J. Dai and D. C. Ludois, "Wireless electric vehicle charging via capacitive power transfer through a conformal bumper," in 2015 IEEE Applied Power Electronics Conference and Exposition (APEC), 2015, pp. 3307-3313.

[6] F. Lu, H. Zhang, H. Hofmann, and C. Mi, "A double-sided LCLC-compensated capacitive power transfer system for electric vehicle charging," IEEE Transactions on Power Electronics, vol. 30, no. 11, pp. 6011-6014, Nov. 2015.

[7] F. Lu, H. Zhang, H. Hofmann, and C. Mi, "A CLLC-compensated high power and large air-gap capacitive power transfer system for electric vehicle charging applications," in 2016 IEEE Applied Power Electronics Conference and Exposition (APEC), 2016, pp. 1721-1725.

[8] F. Lu, H. Zhang, H. Hofmann, and C. Mi, "A double-sided LC-compensation circuit for loosely coupled capacitive power transfer," IEEE Transactions on Power Electronics, vol. 30, no. 2, pp. 1633-1643, Feb. 2018.

[9] F. Lu, H. Zhang, and C Mi, "A two-plate capacitive wireless power transfer system for electric vehicle charging applications," IEEE Transactions on Power Electronics, vol. 33, no. 2, pp. 964-969, Feb. 2018.

[10] H. Y. Zhang, "Load and mutual inductance identification method of ICPT system based on switching capacitors," Chongqing University, 2015.

[11] X. Dai, Y. Sun, and C. Tang, "Dynamic parameters identification method for inductively coupled power transfer system," IEEE International Conference on Sustainable Energy Technologies, Dec. 2010.

[12] Z. H. Wang, Y. P. Li, and Y. Sun, "Load detection model of voltage-fed inductive power transfer system," IEEE Transactions on Power Electronics, vol. 28, no. 11, pp. 5233-5243, Nov. 2013.

[13] J. F. Li, C. L. Liao, and L. F. Wang, "Decoupling method of maximum efficiency and transferring power for electric vehicle wireless charging system via LCCL circuit," Transactions of China Electrotechnical Society, pp. 199-203, 2010.

[14] V. Belevitch, "Summary of the history of circuit theory," Proceeding of the Ire, vol. 50, no. 5, pp.848-855, May 1962.

[15] B. Gustavsen and A. Semlyen, "Rational approximation of frequency domain responses by vector fitting," IEEE Transactions on Power Delivery, vol. 14, no. 3, pp. 1052-1061, Jul. 1999.

[16] W. Cauer, "Die Verwirklichung von Wechselstromwiderständen vor- 
geschriebener Frequenzabhängigkeit," Archiv Für Elektrotechnik, vol. 17, no. 4, pp. 355-388, Apr. 1926

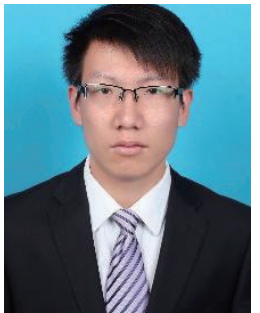

Chen Chen received the B.E. degree in automation from Tianjin Polytechnic University, Tianjin, China, in 2016. He then completed studies in power electronics and electric drives at the University of Chinese Academy of Sciences, Beijing, China. He is currently working towards the M. S. degree in power electronics and electric drives at the Institute of Electrical Engineering, Chinese Academy of Sciences, Beijing, China. His research topic focuses on wireless power transfer for electric vehicle charging and aided inertial navigation system for vehicles.

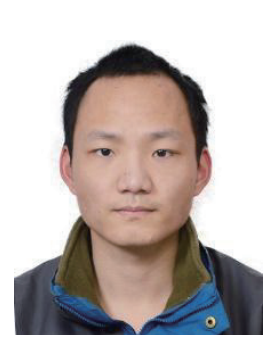

Chenhui Li received the B.E. degree in automation from Tsinghua University, Beijing, China, in 2015. He received the M.S. degree in power electronics and electric drives at the Institute of Electrical Engineering, Chinese Academy of Sciences, Beijing, China, in 2018. His research topic focuses on wireless power transfer for electric vehicle charging. $\mathrm{He}$ is working on the high power capacitive power transfer through air-gap distance and measurement technology of the wireless power transfer system.

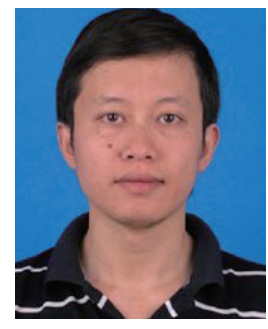

Chenglin Liao received the Ph.D. degree in power machinery and engineering from Beijing Institute of Technology, Beijing, China, in 2001. He then spent two years as a Postdoctoral Researcher with Tsinghua University, Beijing, China. He is currently the Deputy Director of the Department of Vehicle Energy System and Control Technology, Institute of Electrical Engineering, Chinese Academy of Sciences, Beijing, China. He had been involved in research on battery management systems, vehicle control, and wireless charging systems for electric vehicles for the past nine years at the Institute of Electrical Engineering. His research interests include the development of high-power wireless charging systems for commercial electric vehicles.

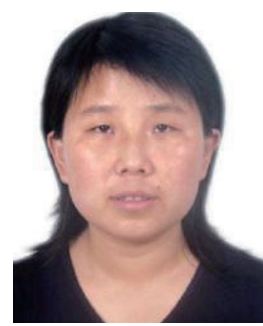

Lifang Wang received the Ph.D. degree in automobile engineering from Jilin University, Jilin, China, in 1997. She then joined the Institute of Electrical Engineering, Chinese Academy of Sciences, Beijing, China. During the Chinese 10th five-year plan (2001-2005), she was a member of the National Specialist Group of the Key Special Electric Vehicle Project of the National 863 Program, and she was the Head of the 863 Special EV Project Office. She is currently the Director of the Department of Vehicle Energy System and Control Technology, Institute of Electrical Engineering, Chinese Academy of Sciences, Beijing, China. She is also the Vice Director of the Key Laboratory of Power Electronics and Electric Drives, Chinese Academy of Sciences. Her research interests include electric vehicle control systems, EV battery management systems, wireless charging systems for EVs, electromagnetic compatibility and smart electricity use. She has directed more than 15 projects in these fields and has published more than 60 papers and 30 patents. 